\title{
PERFECT NULL SETS IN COMPACT HAUSDORFF SPACES
}

\author{
R. B. DARST ${ }^{1}$
}

Our purpose is to establish the following

THEOREM. Suppose $\mu$ is a nontrivial regular nonatomic Borel measure on a compact Hausdorff space $X$. Then $X$ contains a perfect set $P$ such that $\mu(P)=0$.

To this end, we shall use two theorems of W. Rudin [1]:

$\left[\mathrm{R}_{1}\right]$. If $Q$ is a compact Hausdorff space without perfect sets and $f \in C(Q)$, then $f(Q)$ is countable.

$\left[\mathrm{R}_{2}\right]$. If $Q$ is a compact Hausdorff space without perfect sets, $m$ is a regular Borel measure on $Q$ and if $m(E)=0$ for every set $E$ which consists of a single point, then $m(Q)=0$ (i.e., $m$ vanishes identically).

Proof. By $\left[\mathrm{R}_{2}\right]$, there exists a perfect subset $A$ of $X$. If $\mu(A)=0$, we are through; otherwise, considering the restriction of $\mu$ to $A$, it suffices to suppose that $X$ is perfect. It then follows from a proof of Urysohn's Lemma that there exists a continuous function $f$ from $X$ onto the real interval $[0,1]$. Let $\left\{C_{\lambda}\right\}$ be an uncountable collection of pairwise disjoint closed perfect subsets of $[0,1]$. The inverse images, $X_{\lambda}=f^{-1}\left(C_{\lambda}\right)$, are pairwise disjoint closed subsets of $X$ each of which, by $\left[\mathrm{R}_{1}\right]$, contains a perfect set $P_{\lambda}$. Let $P=P_{\lambda}$ where $\mu\left(P_{\lambda}\right)=0$.

\section{BIBLIOGRAPHY}

1. Walter Rudin, Continuous functions on compact sets without perfect subsets, Proc. Amer. Math. Soc. 8(1957), 39-42.

Purdue University

Received by the editors February 8, 1965.

${ }^{1}$ Supported by National Science Foundation Grant GP 03515. 